\title{
Effects of 6-Month Sitagliptin Treatment on Metabolic Parameters in Diabetic Patients Taking Oral Glucocorticoids: A Retrospective Cohort Study
}

\author{
Hisayuki Katsuyama ${ }^{a}$, Akahito Sako ${ }^{\text {a b }}$, Hiroki Adachi ${ }^{a}$, Hidetaka Hamasaki ${ }^{a}$, Hidekatsu Yanai ${ }^{a}$, b, c
}

\begin{abstract}
Background: There are no guidelines for the treatment of diabetes in patients taking glucocorticoids. We studied to understand the effects of 6-month treatment with sitagliptin on metabolic parameters in diabetic patients taking glucocorticoids.

Methods: We retrospectively picked up patients who had been prescribed sitagliptin for 6 months during the continuous prescription of oral glucocorticoids between October 2010 and October 2013 by a chart-based analysis, and compared the data before the sitagliptin treatment with the data at 6 months after the sitagliptin treatment started.
\end{abstract}

Results: Fifteen patients were eligible for the analyses in our study. The plasma glucose and $\mathrm{HbA} 1 \mathrm{c}$ levels were significantly reduced by the sitagliptin treatment. Furthermore, body weight significantly decreased. We found a significant and inverse correlation between the change in $\mathrm{HbA} 1 \mathrm{c}$ levels and $\mathrm{HbA} 1 \mathrm{c}$ levels at baseline. However, there was no significant correlation between the change in HbA1c levels and the daily glucocorticoid dose at baseline.

Conclusions: The present study demonstrated that sitagliptin significantly reduced plasma glucose, HbA1c and body weight. Further, sitagliptin was more effective to improve glycemic control in patients taking glucocorticoids with higher HbAlc levels, independently of the daily glucocorticoid dose.

Keywords: Body weight; Glucocorticoid; HbA1c; Sitagliptin

\footnotetext{
Manuscript accepted for publication March 19, 2015

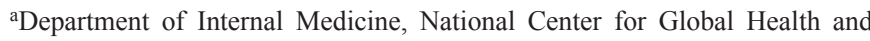
Medicine, Kohnodai Hospital, Chiba 272-8516, Japan

${ }^{\mathrm{b}}$ Clinical Research Center, National Center for Global Health and Medicine, Kohnodai Hospital, Chiba 272-8516, Japan

${ }^{\mathrm{c} C}$ Corresponding Author: Hidekatsu Yanai, Department of Internal Medicine and Clinical Research Center, National Center for Global Health and Medicine, Kohnodai Hospital, 1-7-1 Kohnodai, Chiba 272-8516, Japan.

Email: dyanai@hospk.ncgm.go.jp
}

doi: http://dx.doi.org/10.14740/jocmr2153w

\section{Introduction}

Glucocorticoids are widely prescribed anti-inflammatory and immunosuppressive drugs. Although glucocorticoids provide beneficial effects on inflammatory, allergic, immunologic and malignant diseases, glucocorticoids induce various adverse reactions including osteoporosis, hypertension and hyperglycemia [1-4]. Glucocorticoid-induced insulin resistance and pancreatic islet-cell dysfunction lead to mild increase in fasting plasma glucose levels and a greater increase in postprandial glucose levels [4-9]. The treatment for diabetes in patients taking glucocorticoids may be different from that in diabetic patients without taking glucocorticoids. Although there are no guidelines for the treatment of diabetes in patients taking glucocorticoids, short-acting prandial insulin therapy is currently recommended [10]. Several small studies showed the effectiveness of $\alpha$-glucosidase inhibitors [11], thiazolidinediones [12, 13], and the glucagon-like peptide-1 (GLP-1) receptor agonist [14], for the treatment of diabetes in patients taking glucocorticoids; however, the conclusions led by these studies are premature.

Sitagliptin is one of the dipeptidyl peptidase-4 (DPP-4) inhibitors and is widely used in the treatment of type 2 diabetes. DPP-4 inhibitors enhance levels of active incretin hormones such as the GLP-1 and the glucose-dependent insulinotropic polypeptide (GIP) which are released from the intestinal cells following the meal ingestion [15]. The GLP-1 and GIP stimulate insulin secretion from pancreatic $\beta$ cells and the GLP- 1 inhibits glucagon secretion from pancreatic $\alpha$ cells, which reduces plasma glucose levels. These incretin-based therapies improve postprandial hyperglycemia and have low risk of hypoglycemia due to their glucose-dependent action [16, 17]. Therefore, DPP4 inhibitors may be useful in the management of hyperglycemia in patients with glucocorticoids-induced diabetes, which is characterized by normal or mild increase in fasting plasma glucose levels and a remarkable increase in postprandial glucose levels. However, the evidence is lacking for the effects of DPP-4 inhibitors for these patients. Here, we retrospectively studied effects of 6-month treatment with sitagliptin on metabolic parameters in diabetic patients taking glucocorticoids.

\section{Materials and Methods}

\section{Subjects}

This study was approval by the Institutional Ethics Committee 
Table 1. Baseline Characteristics of Subjects Studied $(n=15)$

\begin{tabular}{|ll} 
Age & $66.9 \pm 13.9$ \\
Sex $(\mathrm{M} / \mathrm{F})$ & $6 / 9$ \\
Body height $(\mathrm{cm})$ & $156 \pm 11$ \\
Body weight $(\mathrm{kg})$ & $57.5 \pm 11.4$ \\
Body mass index $(\mathrm{BMI})\left(\mathrm{kg} / \mathrm{m}^{2}\right)$ & $23.5 \pm 3.0$ \\
Diseases treated by glucocorticoids & \\
\hline Interstitial pneumonia & $2(13 \%)$ \\
Primary multiple myositis & $2(13 \%)$ \\
Polymyalgia rheumatica & $2(13 \%)$ \\
Dermatomyositis & $1(7 \%)$ \\
Rheumatoid arthritis & $1(7 \%)$ \\
Adult-onset Still's disease & $1(7 \%)$ \\
Eosinophilic granulomatosis with polyangitis & $1(7 \%)$ \\
Sarcoidosis & $1(7 \%)$ \\
\hline Panhypopituitarism & $1(7 \%)$ \\
Bullous pemphigoid & $1(7 \%)$ \\
Chronic urticaria & $1(7 \%)$ \\
Bronchial asthma & $1(7 \%)$ \\
\hline
\end{tabular}

in National Center for Global Health and Medicine (NCGMG-001603), and was also performed in accordance with the Declaration of Helsinki. This study was registered with the University Hospital Medical Information Network (UMIN) clinical trials registry, number UMIN 000014508.

We selected patients who had been prescribed sitagliptin for 6 months during the continuous prescription of oral glucocorticoids between October 2010 and October 2013 by a chartbased analysis. Patients with type 1 diabetes were excluded. We also excluded patients who had started taking sitagliptin before the initiation of glucocorticoid therapy, who had taken intravenous glucocorticoids during the study periods, and whose oral glucocorticoid prescription was discontinued during the study period.

\section{Methods}

We compared the data before the sitagliptin treatment with the data at 6 months after the sitagliptin treatment started. Body weight, blood pressure, plasma glucose, HbA1c, serum lowdensity lipoprotein-cholesterol (LDL-C), triglyceride (TG), high-density lipoprotein-cholesterol (HDL-C), aspartate aminotransferase (AST), alanine aminotransferase (ALT) and $\gamma$-glutamyltransferase $(\gamma \mathrm{GTP})$ in studied subjects were measured almost at the same time point before and after 6-month treatment with sitagliptin. LDL-C values were determined by the direct measurement or calculated by using the Friedewald formula. All data are expressed as mean $\pm \mathrm{SD}$.

\section{Statistical analysis}

Comparison of the variables determined before and after the sitagliptin treatment was analyzed by a paired Student's $t$-test. Pearson's simple correlations coefficients were performed to determine the correlations between the data before the start of sitagliptin treatment and changes in $\mathrm{HbA} 1 \mathrm{c}$ after the sitagliptin treatment. $\mathrm{P}<0.05$ was considered to be statistically significant.

\section{Results}

We found 892 patients who had taken sitagliptin between October 2010 and October 2013, and 114 patients had taken oral glucocorticoids at least once during this period. Fifteen patients who had taken sitagliptin for 6 months during the continuous prescription of oral glucocorticoids were eligible for the analyses in our study.

Table 1 shows the baseline characteristics and autoimmune, inflammatory and allergic diseases treated with glucocorticoids. Eleven subjects were diagnosed as glucocorticoid-

Table 2. Other Hypoglycemic Agents Which Subjects Had Taken Before and After the Treatment With Sitagliptin $(n=15)$

\begin{tabular}{lll}
\hline Hypoglycemic agents & Pre-treatment & Post-treatment \\
\hline None & $6(40 \%)$ & $6(40 \%)$ \\
Sulfonylureas & $1(7 \%)$ & $4(27 \%)$ \\
Biguanides & $2(13 \%)$ & $4(27 \%)$ \\
Thiazolidinediones & $3(20 \%)$ & $3(20 \%)$ \\
$\alpha$-glucosidase inhibitors & $5(33 \%)$ & $4(27 \%)$ \\
Glinides & $4(27 \%)$ & $0(0 \%)$ \\
Insulin & $1(7 \%)$ & $1(7 \%)$ \\
\hline
\end{tabular}


Table 3. Changes in Variables After 6-Month Sitagliptin Treatment $(n=15)$

\begin{tabular}{lllll}
\hline & Subjects studied (n) & Pre-treatment & Post-treatment & P value \\
\hline Body weight (kg) & 9 & $61.6(9.9)$ & $58.9(10.6)$ & 0.001 \\
Systolic blood pressure (mm Hg) & 11 & $129(18)$ & $134(17)$ & 0.390 \\
Diastolic blood pressure (mm Hg) & 11 & $72(10)$ & $73(16)$ & 0.914 \\
Plasma glucose (mg/dL) & 15 & $195(72)$ & $121(23)$ & 0.004 \\
HbAlc (\%) & 15 & $7.6(0.9)$ & $6.5(0.9)$ & $<0.001$ \\
LDL-cholesterol (mg/dL) & 9 & $107(24)$ & $100(20)$ & 0.079 \\
Triglyceride (mg/dL) & 11 & $133(55)$ & $163(64)$ & 0.228 \\
HDL-cholesterol (mg/dL) & 8 & $51(16)$ & $52(14)$ & 0.934 \\
AST (IU/L) & 14 & $30(21)$ & $26(13)$ & 0.508 \\
ALT (IU/L) & 14 & $32(28)$ & $24(12)$ & 0.302 \\
$\gamma$ GTP (IU/L) & 8 & $32(12)$ & $31(21)$ & 0.856 \\
eGFR (mL/min/1.73m $\left.{ }^{2}\right)$ & 14 & $86(33)$ & $80(35)$ & 0.029 \\
\hline
\end{tabular}

induced diabetes and four subjects were diagnosed as type 2 diabetes exacerbated by glucocorticoids. The glucocorticoid dose was with the equivalent of $7.8 \pm 6.0 \mathrm{mg} /$ day of prednisolone at baseline, and $5.7 \pm 4.2 \mathrm{mg}$ /day after the sitagliptin treatment with no significant change $(\mathrm{P}=0.191)$. The dose of sitagliptin was $47 \pm 8 \mathrm{mg} / \mathrm{dL}$ at baseline and $55 \pm 24 \mathrm{mg} / \mathrm{dL}$ after the sitagliptin treatment $(\mathrm{P}=0.173)$. Other hypoglycemic agents taken before and after the sitagliptin treatment were shown in Table 2. Five subjects were treated with the sitagliptin monotherapy during the study period.

Table 3 shows the changes in the clinical parameters after the 6-month sitagliptin treatment. The plasma glucose and HbA1c levels were significantly reduced by the sitagliptin treatment. Furthermore, the body weight significantly decreased.

We analyzed the correlation between change in $\mathrm{HbA} 1 \mathrm{c}$ levels and HbAlc levels at baseline. We found a significant and inverse correlation between the change in HbAlc levels and HbAlc levels at baseline (Fig. 1a). However, there was no significant correlation between the change in $\mathrm{HbA} 1 \mathrm{c}$ levels and the daily glucocorticoid dose at baseline (Fig. 1b).

Subgroup analyses were performed in five subjects (two males and three females) treated with the sitagliptin monotherapy to exclude the influence of other hypoglycemic agents. Four subjects were diagnosed as glucocorticoid-induced diabetes and one patient was diagnosed as having type 2 diabetes. The mean \pm SD of age and BMI were $54.7 \pm 12.8$ years old and $23.5 \pm 2.8 \mathrm{~kg} / \mathrm{m}^{2}$, respectively. Table 4 shows the changes in the clinical and biochemical parameters after the 6-month sitagliptin monotherapy in these subjects. The HbAlc levels also significantly decreased after the sitagliptin treatment by $1.04 \%$, and plasma glucose levels showed a tendency to decrease. Body weight also showed a tendency to decrease by $3.5 \mathrm{~kg}$.

\section{Discussion}

The present study demonstrated that the sitagliptin treatment including the add-on therapy and monotherapy significantly
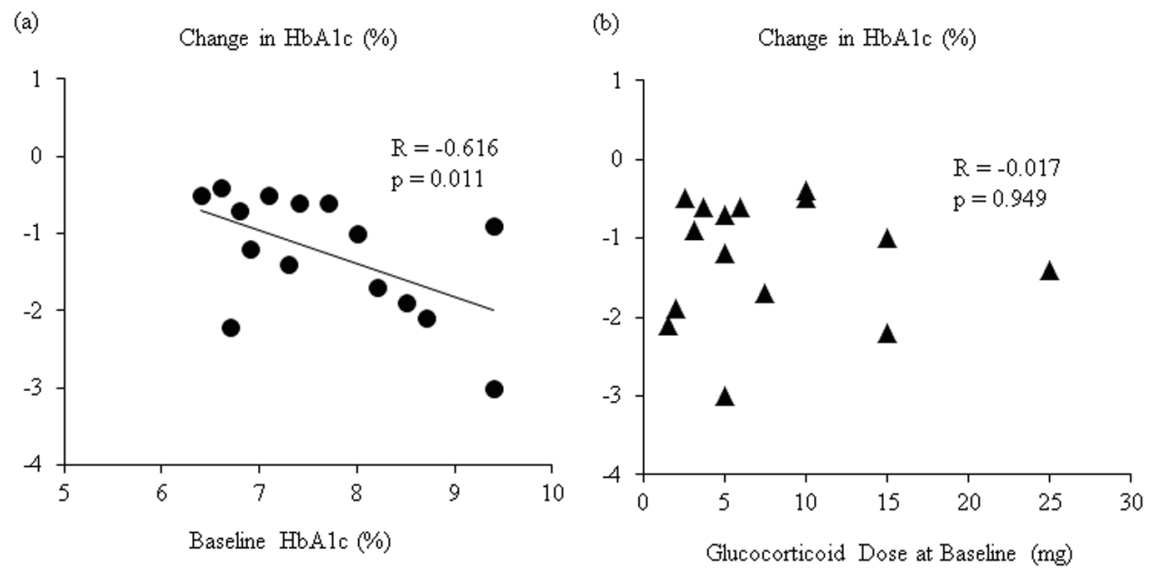

Figure 1. (a) Correlation between changes in $\mathrm{HbA} 1 \mathrm{c}$ after 6-month sitagliptin treatment and $\mathrm{HbA} 1 \mathrm{c}$ levels at baseline. (b) Correlation between changes in $\mathrm{HbA} 1 \mathrm{c}$ after 6-month sitagliptin treatment and daily glucocorticoids dose at baseline. 
Table 4. Changes in Variables After 6-Month Sitagliptin Monotherapy $(n=5)$

\begin{tabular}{lllll}
\hline & Subjects studied (n) & Pre-treatment & Post-treatment & P value \\
\hline Body weight (kg) & 3 & $64.6(3.6)$ & $61.1(2.5)$ & 0.087 \\
Systolic blood pressure (mm Hg) & 4 & $126(12)$ & $133(17)$ & 0.568 \\
Diastolic blood pressure (mm Hg) & 4 & $75(4)$ & $83(10)$ & 0.345 \\
Plasma glucose (mg/dL) & 5 & $192(76)$ & $107(22)$ & 0.060 \\
HbA1c (\%) & 5 & $7.0(0.3)$ & $5.9(0.7)$ & 0.036 \\
LDL-cholesterol (mg/dL) & 2 & $135(4)$ & $116(18)$ & 0.465 \\
Triglyceride (mg/dL) & 4 & $131(44)$ & $128(36)$ & 0.957 \\
HDL-cholesterol (mg/dL) & 3 & $59(16)$ & $48(11)$ & 0.148 \\
AST (IU/L) & 5 & $30(16)$ & $28(19)$ & 0.789 \\
ALT (IU/L) & 5 & $30(17)$ & $26(14)$ & 0.710 \\
$\gamma$ GTP (IU/L) & 3 & $39(11)$ & $35(24)$ & 0.789 \\
eGFR (mL/min/1.73 m $\left.{ }^{2}\right)$ & 5 & $111(36)$ & $108(40)$ & 0.486 \\
\hline
\end{tabular}

improved HbA1c levels, indicating the effectiveness of sitagliptin for the treatment of diabetes in patients taking glucocorticoids. Glucocorticoids have been reported to show the dosedependent effect on glucose metabolism [10]. However, our study showed that the sitagliptin treatment improved $\mathrm{HbAlc}$ levels, independently of the daily glucocorticoid dose, which also supported the usefulness of sitagliptin for the treatment of diabetes in patients taking glucocorticoids.

The underlying mechanisms for glucocorticoid-induced hyperglycemia include increased hepatic endogenous glucose production, reduced insulin-stimulated glucose uptake in skeletal muscle, increased visceral fat deposition and insulin resistance $[4,5,18]$. Moreover, glucocorticoids may impair insulin secretion from $\beta$ cells and may augment glucagon secretion from $\alpha$ cells $[4,6,7]$. Since glucocorticoids usually cause mild increase in fasting plasma glucose and a greater increase in postprandial glucose, rapid-acting prandial insulin therapy without basal insulin is currently recommended [10]. However, insulin therapy increases the risk of adverse effects, such as hypoglycemia and weight gain. Furthermore, it is too difficult to determine the optimal insulin dose, because the dose of glucocorticoids is frequently changed due to the severity of the diseases treated by glucocorticoids.

Several small studies showed the effectiveness of oral hypoglycemic agents. Tanaka et al reported the treatment for steroid-induced diabetes with $\alpha$-glucosidase inhibitor, voglibose (1 day). They treated six patients and showed a decrease in the amount of urinary glucose [11]. Willi et al evaluated the efficacy of a thiazolidinedione, 5 - 8 weeks troglitazone treatment, in seven patients with long-standing steroid-induced diabetes, and they found that troglitazone significantly reduced $\mathrm{HbA} 1 \mathrm{c}$ from $7.8 \pm 0.4 \%$ to $7.2 \pm 0.4 \%$ [12]. Morita et al studied the effect of troglitazone treatment for dexamethasoneinduced glucose intolerance in five healthy men, and showed that the 2-week troglitazone administration reduced the 3-day dexamethasone administration-induced increase of the mean area under the curve from 0 to $3 \mathrm{~h}$ for both plasma glucose and serum insulin concentrations during a 75 -g oral glucose toler- ance test [13]. These studies include premature factors such as monitor of diabetes [11], a short study period and small number of participants [11-13].

Recently, van Raalte et al performed a randomized, placebo-controlled, double-blind, crossover study in eight healthy men, to understand whether treatment with the GLP-1 agonist, exenatide could prevent glucocorticoids-induced glucose intolerance. Participants received three therapeutic regimens for two consecutive days: 1) $80 \mathrm{mg}$ of oral prednisolone everyday and intravenous exenatide infusion; 2) $80 \mathrm{mg}$ of oral prednisolone and intravenous saline infusion; and 3) oral placeboprednisolone and intravenous saline infusion. Oral prednisolone and intravenous saline infusion increased postprandial glucose levels, which was prevented by the concomitant infusion of exenatide. Exenatide reduced prednisolone-induced hyperglucagonemia during the meal challenge. Oral prednisolone and intravenous saline infusion decreased the firstphase glucose- and arginine-stimulated C-peptide secretion, whereas oral prednisolone and intravenous exenatide infusion improved the first- and second-phase glucose- and argininestimulated C-peptide secretion, suggesting the effectiveness of the incretin-based therapy for the prevention of steroid diabetes [14]. However, this is the study to report acute effect of the incretin-based therapy for steroid-induced glucose intolerance in healthy individuals.

Jensen et al reported that glucocorticoid-induced glucose intolerance is associated with a progressive decline of incretin effects [19], suggesting the incretin-based therapy including the DPP-4 inhibitors may be useful for the treatment of glucocorticoid-induced diabetes. We previously reported a patient with glucocorticoid-induced diabetes whose glucose levels were ameliorated by the use of DPP-4 inhibitor, sitagliptin [20]. Ohashi et al reported that alogliptin improved steroid-induced hyperglycemia by decrease of glucagon levels through an increase in plasma GLP-1 levels, in 11 patients with chronic kidney disease. The mean \pm SD of alogliptin treatment periods was $11.4 \pm 12.8$ days [21]. van Genugten et al studied effects of the co-administration of daily $100 \mathrm{mg}$ sitagliptin with daily 30 
mg prednisolone for 2 weeks in men with the metabolic syndrome. However, sitagliptin could not prevent prednisoloneinduced increment in postprandial glucose concentrations [22]. To our knowledge, our study is the first to report chronic effect (6 months) of the DPP-4 inhibitor on glucose metabolism including $\mathrm{HbA} 1 \mathrm{c}$, in patients with long-standing steroid-induced diabetes. The present study also demonstrated a significant and inverse correlation between changes in $\mathrm{HbAlc}$ levels and $\mathrm{HbA} 1 \mathrm{c}$ levels at baseline, suggesting that sitagliptin is more effective to improve glycemic control with glucocorticoidtreated patients with poorer glucose control.

Glucocorticoids usually induce weight gain [23]; however, our study showed that the sitagliptin treatment significantly reduced body weight by $2.7 \mathrm{~kg}$. In our previous study, sitagliptin also significantly reduced body weight after the 6-month treatment in patients with type 2 diabetes, and a significant and negative correlation between change in body weight and body mass index at baseline was observed [24]. Sitagliptin may reduce body weight by ameliorating obesity-related factors such as insulin resistance. However, the underlying mechanism for sitagliptin-mediated reduction of body weight in patients taking glucocorticoids remains unknown, which should be elucidated in the future.

The present study has several limitations. First, other hypoglycemic agents, food intakes and/or exercise levels may have an influence on the study results. Second, we could not exclude the influence of diseases treated with glucocorticoids. Third, our analysis included both patients with glucocorticoidinduced diabetes and patients with pre-existing type 2 diabetes. The number of studied subjects was small. Further studies, preferably with larger numbers of subjects, will be needed.

In conclusion, we studied effects of 6-month sitagliptin treatment on metabolic parameters in diabetic patients taking oral glucocorticoids, and found that sitagliptin significantly reduced plasma glucose, $\mathrm{HbA1c}$ and body weight. In the present study, sitagliptin is more effective to improve glycemic control in diabetic patients taking glucocorticoids with higher $\mathrm{HbA1c}$ levels, independently of the daily glucocorticoid dose.

\section{Acknowledgement}

The authors would like to thank Tomoko Kaga and colleagues in Clinical Research Center, National Center for Global Health and Medicine Kohnodai Hospital, for their technical help.

\section{Conflict of Interests}

The authors declare that they have no conflicts of interest concerning this article.

\section{Funding Support}

This work was supported by Grants-in-Aid for Research from the National Center for Global Health and Medicine (26A201).

\section{References}

1. Weinstein RS. Glucocorticoid-induced osteonecrosis. Endocrine. 2012;41(2):183-190.

2. Whitworth JA. Adrenocorticotrophin and steroid-induced hypertension in humans. Kidney Int Suppl. 1992;37:S3437.

3. Hwang JL, Weiss RE. Steroid-induced diabetes: a clinical and molecular approach to understanding and treatment. Diabetes Metab Res Rev. 2014;30(2):96-102.

4. van Raalte DH, Diamant M. Steroid diabetes: from mechanism to treatment? Neth J Med. 2014;72(2):62-72.

5. Rockall AG, Sohaib SA, Evans D, Kaltsas G, Isidori AM, Monson JP, Besser GM, et al. Computed tomography assessment of fat distribution in male and female patients with Cushing's syndrome. Eur J Endocrinol. 2003;149(6):561-567.

6. van Raalte DH, Brands M, van der Zijl NJ, Muskiet MH, Pouwels PJ, Ackermans MT, Sauerwein HP, et al. Lowdose glucocorticoid treatment affects multiple aspects of intermediary metabolism in healthy humans: a randomised controlled trial. Diabetologia. 2011;54(8):21032112.

7. Wise JK, Hendler R, Felig P. Influence of glucocorticoids on glucagon secretion and plasma amino acid concentrations in man. J Clin Invest. 1973;52(11):2774-2782.

8. Iwamoto T, Kagawa Y, Naito Y, Kuzuhara S, Kojima M. Steroid-induced diabetes mellitus and related risk factors in patients with neurologic diseases. Pharmacotherapy. 2004;24(4):508-514.

9. Burt MG, Roberts GW, Aguilar-Loza NR, Frith P, Stranks $\mathrm{SN}$. Continuous monitoring of circadian glycemic patterns in patients receiving prednisolone for COPD. J Clin Endocrinol Metab. 2011;96(6):1789-1796.

10. Clore JN, Thurby-Hay L. Glucocorticoid-induced hyperglycemia. Endocr Pract. 2009;15(5):469-474.

11. Tanaka M, Endo K, Suzuki T, Maruyama Y, Kondo A. Treatment for steroid-induced diabetes with alpha-glucosidase inhibitor, voglibose. Eur J Neurol. 1998;5(3):315.

12. Willi SM, Kennedy A, Brant BP, Wallace P, Rogers NL, Garvey WT. Effective use of thiazolidinediones for the treatment of glucocorticoid-induced diabetes. Diabetes Res Clin Pract. 2002;58(2):87-96.

13. Morita H, Oki Y, Ito T, Ohishi H, Suzuki S, Nakamura H. Administration of troglitazone, but not pioglitazone, reduces insulin resistance caused by short-term dexamethasone (DXM) treatment by accelerating the metabolism of DXM. Diabetes Care. 2001;24(4):788-789.

14. van Raalte DH, van Genugten RE, Linssen MM, Ouwens DM, Diamant M. Glucagon-like peptide-1 receptor agonist treatment prevents glucocorticoid-induced glucose intolerance and islet-cell dysfunction in humans. Diabetes Care. 2011;34(2):412-417.

15. Drucker DJ. The biology of incretin hormones. Cell Metab. 2006;3(3):153-165.

16. Drucker DJ, Nauck MA. The incretin system: glucagon-like peptide-1 receptor agonists and dipeptidyl peptidase-4 inhibitors in type 2 diabetes. Lancet. 
2006;368(9548):1696-1705.

17. Ahren B. Use of DPP-4 inhibitors in type 2 diabetes: focus on sitagliptin. Diabetes Metab Syndr Obes. 2010;3:31-41.

18. Rizza RA, Mandarino LJ, Gerich JE. Cortisol-induced insulin resistance in man: impaired suppression of glucose production and stimulation of glucose utilization due to a postreceptor detect of insulin action. J Clin Endocrinol Metab. 1982;54(1):131-138.

19. Jensen DH, Aaboe K, Henriksen JE, Volund A, Holst JJ, Madsbad S, Krarup T. Steroid-induced insulin resistance and impaired glucose tolerance are both associated with a progressive decline of incretin effect in first-degree relatives of patients with type 2 diabetes. Diabetologia. 2012;55(5):1406-1416.

20. Yanai H, Masui Y, Yoshikawa R, Kunimatsu J, Kaneko H. Dipeptidyl peptidase-4 inhibitor for steroid-induced diabetes. World J Diabetes. 2010;1(3):99-100.

21. Ohashi N, Tsuji N, Naito Y, Iwakura T, Isobe S, Ono M, Fujikura T, et al. Alogliptin improves steroid-induced hyperglycemia in treatment-naive Japanese patients with chronic kidney disease by decrease of plasma glucagon levels. Med Sci Monit. 2014;20:587-593.

22. van Genugten RE, van Raalte DH, Muskiet MH, Heymans MW, Pouwels PJ, Ouwens DM, Mari A, et al. Does dipeptidyl peptidase-4 inhibition prevent the diabetogenic effects of glucocorticoids in men with the metabolic syndrome? A randomized controlled trial. Eur J Endocrinol. 2014;170(3):429-439.

23. Huscher D, Thiele K, Gromnica-Ihle E, Hein G, Demary W, Dreher R, Zink A, et al. Dose-related patterns of glucocorticoid-induced side effects. Ann Rheum Dis. 2009;68(7):1119-1124.

24. Yanai H, Adachi H, Hamasaki H, Masui Y, Yoshikawa R, Moriyama S, Mishima S, et al. Effects of 6-month sitagliptin treatment on glucose and lipid metabolism, blood pressure, body weight and renal function in type 2 diabetic patients: a chart-based analysis. J Clin Med Res. 2012;4(4):251-258. 